\title{
Analysis of the construction of the high- temperature gas infrared radiator with the use of virtual prototyping
}

\author{
Anton N. Ermolaev ${ }^{1}$, and Olga V. Khaustova ${ }^{2, *}$ \\ ${ }^{1}$ Tyumen industrial University, 625000 Tyumen, Russia \\ ${ }^{2}$ National Research Tomsk Polytechnic University, 634050 Tomsk, Russia
}

\begin{abstract}
Method of virtual prototyping with the following mathematical modeling was used to simulate the heat-mass exchange and combustion during the operation of high-temperature gas infrared radiators, and to find optimal technical solutions for its design. The most authoritative and approved software product Ansys Multiphysics was used. The results of the mathematical modeling of heat and mass transfer in a turbulent reaction medium with combustion reproduce the experimental data produced by a measurement in real operating conditions of the gas-fired infrared heat emitter. The temperature distribution along the height of the ceramic nozzle was established. Obtained results enable estimation of the ignition and combustion zones.
\end{abstract}

\section{Introduction}

Two acute tasks of energy resources saving and energy efficiency increasing can be achieved by replacing convective heating systems of production buildings, warehouses, large pavilions, hangars with radiant heating systems based on high-temperature gas infrared radiators $[1,2]$. Over many years of operation, high-temperature gas infrared radiators has proven itself as a reliable, energy-efficient and environmentally friendly source of converting fuel energy into thermal energy. The conversion factor of such heating devices is $98 \%$ [1], but the fraction of the radiative heat flux transferred to the work zone by radiation is only $58-61 \%$ [2]. The rest of the heat energy is accounted for heat losses with outgoing gases $(24-30 \%)$ and the casing of the radiator $(9-18 \%)$. The analysis of the high-temperature gas infrared radiators design aimed to improve the effectiveness of its work is an up-to-date practice [2].

Typical model of high-temperature gas infrared radiators consists of a number of complex technical elements (Figure 1) and includes a large number of variable parameters. In the 21 st century, the technology of virtual prototyping is increasingly used [3-6]. It allows to analyze all the mechanisms of production at the design stage, look inside the technical process, identify design errors, and make pre-operational evaluation of the

\footnotetext{
* Corresponding author: olgavguk@tpu.ru
} 
efficiency with less labor. In other words, a virtual prototype is a digital twin or a preliminary digital assembly of a technological product at the stage of designing.

Therefore, the method of virtual prototyping with the following mathematical modeling was used to simulate the heat-mass exchange and combustion during the operation of hightemperature gas infrared radiators, and to find optimal technical solutions for its design. The most authoritative and approved software product Ansys Multiphysics was adopted as the research tool [4-6].

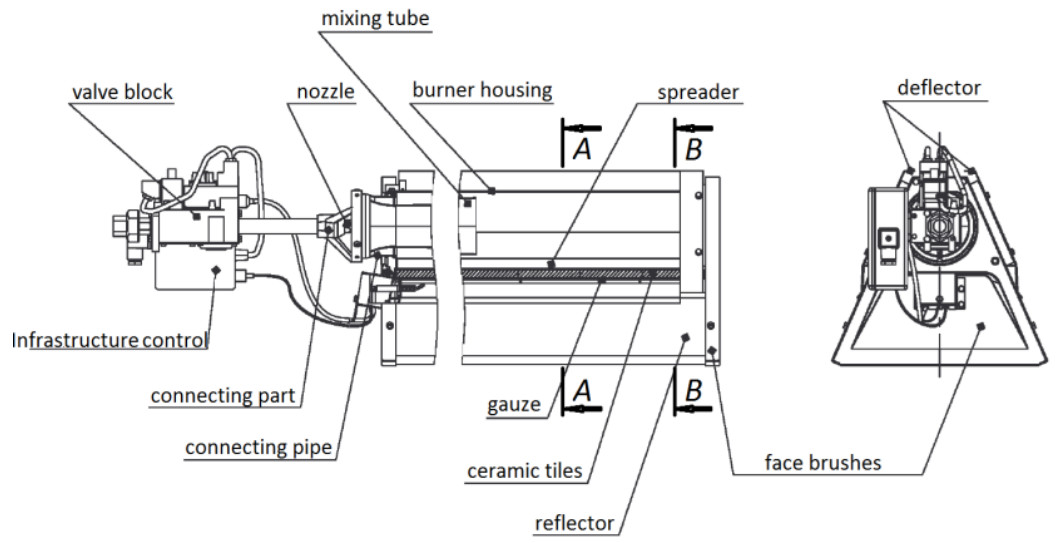

Fig. 1. Typical model of high-temperature gas infrared radiator.

\section{Results of the study}

Full virtual prototype of high-temperature gas infrared burner was built based on its sketches and complete geometric model (Fig. 1). The construction of the computational grid is performed by ANSYS Workbench, namely, the volume is divided into finite elements - tetrahedrons in the amount of $3 \cdot 10^{6}$. Boundary conditions are: the material of the radiator construction is $18-8$ steel with a thickness of $0.002 \mathrm{~m}$; fuel - natural gas $(\rho=0.784$ $\mathrm{kg} / \mathrm{m}^{3}, \mathrm{Q}=37.26 \mathrm{MJ} / \mathrm{kg}$ ); oxidizer - air oxygen; fuel mass flow rate $-0.5 \mathrm{~m}^{3}$ / hour; air fuel ratio is $10.374 \mathrm{~m}^{3} / \mathrm{m}^{3}$. The internal air temperature is assumed to be $+20^{\circ} \mathrm{C}$. Process is in a steady state.

Using ANSYS Fluent, the following processes and phenomena were modeled: combustion of natural gas, turbulence, convective, conductive and radiant heat exchange. To describe the ongoing chemical reaction, a kinetic model of laminar combustion based on a simplified kinetic scheme of the gross reactions of methane oxidation was adopted. Calculation of radiant heat exchange was performed using the Discrete Ordinates radiation model. The validity of the selected mathematical models is confirmed by full-scale tests [1, $2,6]$.
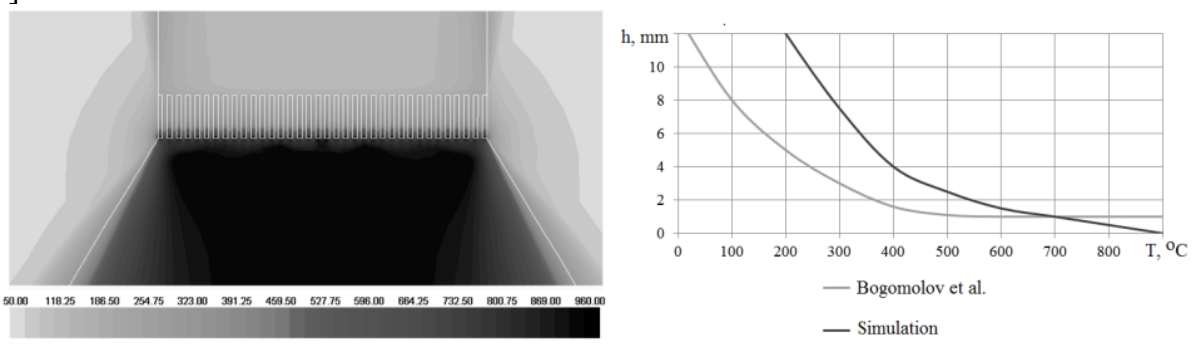

Fig. 2. Temperatures contours $\left({ }^{\circ} \mathrm{C}\right)$ in gas-fired infrared heat emitter (a) and comparison with the experimental data (b) of [1]. 
The results of the mathematical modeling of heat and mass transfer in a turbulent reaction medium with combustion reproduce the experimental data produced by a measurement in real operating conditions of the gas-fired infrared heat emitter (see Fig. 2). According to the designed virtual prototype, the temperature of the ceramic tiles (see Fig. 1) for all investigated emitters was $900{ }^{\circ} \mathrm{C}$ (Fig. 2). In the course of analysis of the designed virtual prototype of high-temperature gas infrared burner, a dependence describing the temperature distribution along the height of the ceramic nozzle was established. The graph in Figure 2 demonstrates a qualitative agreement in mechanisms of combustion initiation and the location of the kinetic combustion region between the virtual prototype of the gasfired infrared heat emitter and the experiment [1]. The numerical difference in the values of the temperatures at the entrance to the perforation of the ceramic nozzle is explained by the design and regime characteristics of the radiators [1,2].

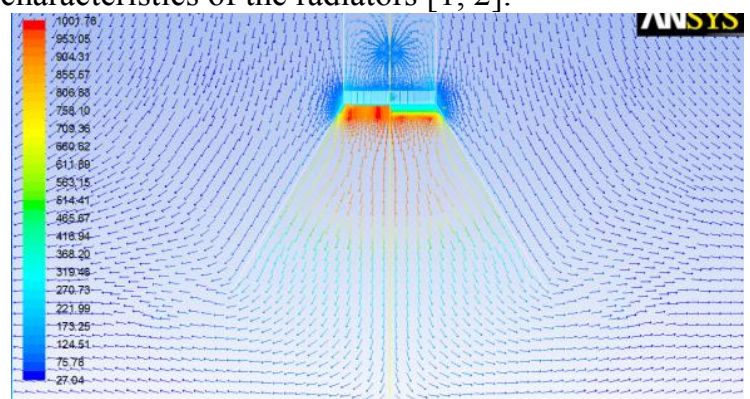

\section{Section A-A (see fig. 1) Section B-B (see fig. 1)}

Fig. 3. Vectors colored by temperature $\left({ }^{\circ} \mathrm{C}\right)$ in the longitudinal section of the ceramic tiles of the gasfired infrared heat emitter.

Detailed temperature fields and vectors in the longitudinal section of the ceramic tiles of the gas-fired infrared heat emitter (Fig. 3) enable estimation of the ignition and combustion zones. The ignition takes place at the outlet of the ceramic tiles, because fuel is premixed with air in mixing tube (see Fig. 1) before entering the holes in the ceramic tile. As a result narrow laminar combustion front moves through holes of ceramic tiles, and has its maximum at the outlet of the cylindrical holes.

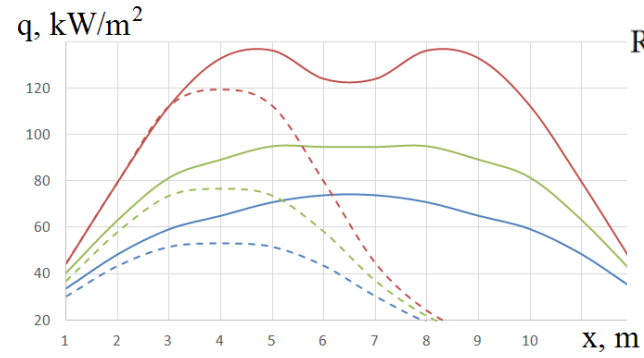

a)

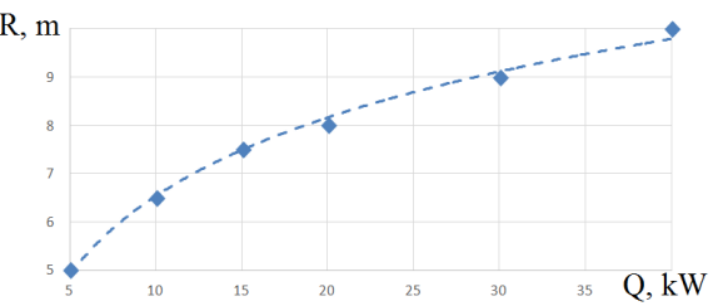

b)

Fig. 4. The distribution of the irradiation intensity (a) and diameter of $10 \mathrm{~W} / \mathrm{m}^{2}$ isosurface in the working zone.

To verify the fulfillment of thermal comfort conditions, the distribution of the irradiation intensity in the working zone was calculated depending on the parameters studied. The maximum intensity of infrared irradiation of the workplace should not exceed $150 \mathrm{~W} / \mathrm{m}^{2}$. For the parameters studied, this condition corresponds to the following heights of the virtual prototypes of high-temperature gas infrared burner suspension: $5 \mathrm{~kW}-4 \mathrm{~m}$ $\left(119.3 \mathrm{~W} / \mathrm{m}^{2}\right) ; 10 \mathrm{~kW}-6 \mathrm{~m}\left(106.1 \mathrm{~W} / \mathrm{m}^{2}\right) ; 15 \mathrm{~kW}-7 \mathrm{~m}\left(116.9 \mathrm{~W} / \mathrm{m}^{2}\right) ; 20 \mathrm{~kW}-8 \mathrm{~m}(119.3$ $\left.\mathrm{W} / \mathrm{m}^{2}\right) ; 30-10 \mathrm{~kW}\left(114.6 \mathrm{~W} / \mathrm{m}^{2}\right)$. For the virtual prototype of high-temperature gas 
infrared burner with a power of $40 \mathrm{~kW}$ this condition is violated $\left(152.8 \mathrm{~W} / \mathrm{m}^{2}\right)$ when placed at a maximum height of $10 \mathrm{~m}$. Dependencies have been established, indicating that the higher the level of high-temperature gas infrared burner suspension results in the more uniform the distribution of local heat fluxes in the working zone and the lower their amplitude (Fig. 4, a). The impact of the high-temperature gas infrared burner thermal power on the diameter of $10 \mathrm{~W} / \mathrm{m}^{2}$ isosurface in the working zone is close to the logarithmic (Fig. $4, b)$.

\section{Short conclusion}

Virtual prototype of high-temperature gas infrared burner has been designed to search for a technical solution in accordance with the chosen criterion of optimality and check the efficiency and effectiveness of such solutions with less labor and investment.

Optimization of the design will be achieved by sequential refinement of the virtual prototype of the radiator. With an increase in the degree of detail of virtual prototyping, a more precise structural and functional imitation will become possible. The ideal situation will be reached when the virtual prototype will have the whole set of characteristics of the physical prototype. Thus, virtual prototyping eliminates the need for expensive and laborintensive physical prototypes.

Using the virtual prototyping it was estimated that the most uniform intensity of irradiation of the working zone is ensured when the high-temperature gas infrared burner is installed of the same power at a distance shown in fig. 4 . The value of heat flows for such a heating system organization is $25 \%$ higher than when a single high-temperature gas infrared burner located at a greater distance.

\section{References}

1. A.I. Bogomolov, D. Ya. Vigdorchik, M.A. Maevsky, Gas burners of infrared radiation and their application (Stroiizdat, Moscow, 1967)

2. A. N. Ermolaev, S. A. Khaustov, EPJ Web Conf., 110, 01038 (2017)

3. A. V. Kazakov, R. B. Tabakaev, P. Y. Novoseltsev, A. V. Astafev, A.V., MATEC Web Conf., 19, 01014 (2014)

4. S. A. Khaustov, A. S. Zavorin, K. V. Buvakov, L. D. Kudryashova, A. V. Tshelkunova, EPJ Web Conf., 82, 01041 (2015)

5. S. A. Khaustov, A. S. Zavorin, K. V. Buvakov, V. A. Sheikin, EPJ Web Conf., 82, 01039 (2015)

6. S. A. Khaustov, Y. A. Belousova, K. V. Buvakov, A. Y. Dolgih, R. N. Kulesh. Proc. of 11th International Forum on Strategic Technology (IFOST - 2016), 2, 548-551 (2016)

7. Sanitary rules and regulations 2.2.4.548-96. 2.2.4 Physical factors of the production environment. Hygienic requirements for the microclimate of production facilities (Information and Publishing Center of the Ministry of Health of Russia, Moscow, 1997)

8. Code of Regulations 60.13330.2012. Heating, ventilation and air conditioning (Ministry of Regional Development of Russia, Moscow 2012) 